ISBN: 978-1-948012-12-6

\title{
DESIGN OF MICRO POWER LIQUID LEVEL TRANSMITTER BASED ON C8051F410 CHIP SYSTEM
}

\author{
Liu Qi1 ${ }^{1}$ Li Baohua ${ }^{1}$, Wang Ying ${ }^{1 *}$, Li Shuo ${ }^{1}$, Liang Kai' ${ }^{1}$ Liu Jinran ${ }^{2}$ \\ ${ }^{1}$ Jilin Institute of Chemical Technology, Chengde street, Jilin, China \\ ${ }^{2}$ No. 2 Jihua Primary School, Yingkou road, Jilin, China \\ *Corresponding Author Email: wangying20002@sina.com
}

This is an open access article distributed under the Creative Commons Attribution License, which permits unrestricted use, distribution, and reproduction in any medium, provided the original work is properly cited

\section{ARTICLE DETAILS}

\section{Article History:}

Received 26 June 2018

Accepted 2 July 2018

Available online 1 August 2018

\section{ABSTRACT}

This paper introduces a method for designing a micropower smart level transmitter based on the C8051F410 system-on-chip. The internal AD/DA module is combined with peripheral circuit to complete the collection of voltage and transmission of 4-20mA current. And LCD segment liquid crystal is driven directly by MCU pin to display the current level. And Bubble filtering is used to stabilize the sampling and transmission values. This micro power system is powered by $24 \mathrm{~V}$ DC power supply and its working current is under $2 \mathrm{~mA}$. The system has two ranges $(0-$ $20 \mathrm{~cm}, 0-50 \mathrm{~cm}$ ), and gives the actual measured data records of the two ranges. It also analyzes the causes of measurement errors and offers corresponding improvement measures. The design scheme of the system has some reference for designing industrial instrument.

\section{KEYWORDS}

System on a chip, micro power consumption, transmitter.

\section{INTRODUCTION}

More and more designers are paying attention to power consumption with the urgent demand for low power consumption. The traditional way to reduce power consumption is mainly done by hardware [1]. In this paper, two key aspects of the software and hardware design of the micro power level transmitter are studied. The designed system takes 410-chip system as control unit. This can give full play to the characteristics of both hardware and software. It directly displays the level on the premise of micro power consumption and converts the analog of liquid level to electrical signal of $4 \sim 20 \mathrm{~mA}$ and sends it to control room in the meanwhile. This method can be used for reference in the design of micro power meter.

\subsection{Scheme principle}

The principle of the scheme is shown in Figure 1.

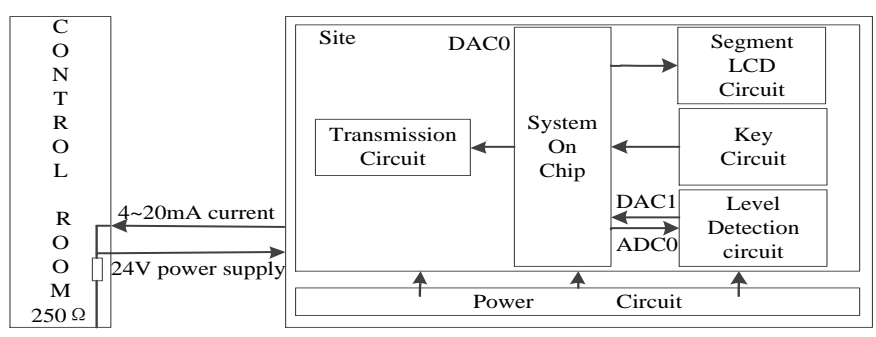

Figure 1: Schematic diagram

This design is based on the 410-chip system including liquid level display circuit, segment liquid crystal display circuit, converter circuit, key circuit, etc. Liquid crystal display circuit and key circuit are used to achieve manmachine information exchange; liquid level detection circuit is used to measure current level depth; converter circuit is used to transfer current signal; and key circuit is used to switch the range. The microcontroller converts the current measured voltage value to the depth of liquid leve and sends it to the display and controls the output of DACO according to the current voltage value. The output signal is transformed into the corresponding electrical signal through the transformation circuit and sent to the control room. It can also be converted to $1 \sim 5 \mathrm{~V}$ voltage if the control room is connected with $250 \Omega$ resistor [2].

\section{HARDWARE CIRCUIT DESIGN}

\subsection{Minimum system and converter circuit}

The C8051F410 device is a low-power mixed-signal system-on-chip MCU. Some of the key features are listed below: analog multiplexer, two 12-bit current output DACs, high-precision programmable $24.5 \mathrm{MHz}$ internal oscillator, four 16-bit timers, twenty-four I/O ports, etc. The user software can completely control the peripherals, and it can also turn off any peripherals to save power. In the industrial temperature range ($40^{\circ} \mathrm{C} \sim+85^{\circ} \mathrm{C}$ ), each device can operate at $2.0 \sim 2.75 \mathrm{~V}$ voltage (the voltage of power supply can reach $5.25 \mathrm{~V}$ when the on-chip voltage regulator is used). C8051F410 is packaged in LQFP having 32 pins [3]. Figure 2 is the minimum system circuit diagram for C8151F410.

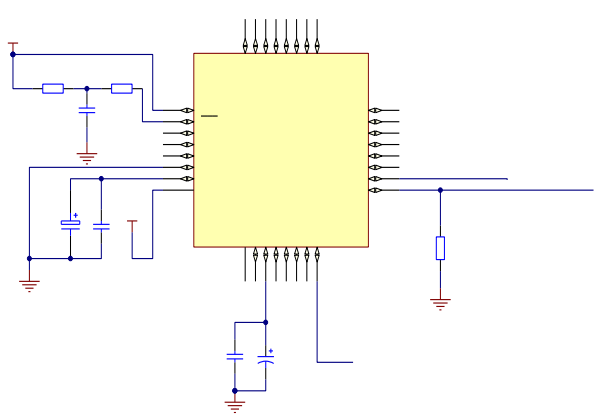

Figure 2: Minimum system circuit diagram 


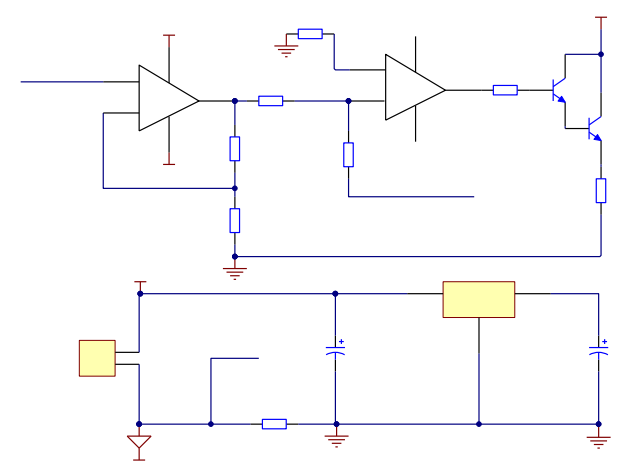

Figure 3: Converter circuit

The converter circuit is shown in Figure 3 and composed of amplifier adjustment circuit, transistor current compensation circuit and so on. In the figure, DAC0 is the voltage input. When the input voltage is $0.75 \mathrm{~V}$ (maximum), the output of the A operational amplifier is 1.5V. Because of the virtual fault, the non-inverting input of B-circuit operational amplifier is $0 \mathrm{~V}, \mathrm{Text} 1$ is $-1.5 \mathrm{~V}$. The output of the B operational amplifier controls the switch of the triode so as to compensate for the current. The current that flows through $75 \Omega$ resistors is $4 \sim 20 \mathrm{~mA}$. However, it should be noted that the lowest input of DAC0 in the figure cannot be 0V [4]. At this point, the transistor will be in the off-state so that it cannot compensate for the current. This problem is solved by programming so that the lowest output of DAC0 can maintain the operation of the entire transmission circuit.

\subsection{Liquid level detection circuit}

The ADC conversion voltage range of the C8051F410 is $0 \sim 2.2 \mathrm{~V}$, and the minimum current of the DAC output is $0.25 \mathrm{~mA}$. When the resistance value is $8.8 \mathrm{k} \Omega$, the voltage value reaches $2.2 \mathrm{~V}$ which is the maximum value of the ADC sampling. The smaller the resistance of each selected resistor, the higher the resolution of the detection circuit [5]. In this design, we select 88 resistors that are $100 \Omega$. There is magnetic suspension ball on the outside of the white steel tube. The position of the floating ball changes with the variation of liquid level. The reed switches are turned on when they are attracted by the magnet. Therefore, it can measure different depths of liquid level. Ideally, the magnetic properties of a suspended ball will only allow one reed switch to conduct, and this is the most accurate test result at this time. Due to the limited conditions, The magnetic properties of our floating ball can make two adjacent reed pipes turn on as long as the resolution of the liquid level detection circuit (reed switch) is sufficiently high (more), which has little effect on the detection result and in the range of error.

\subsection{Segment LCD circuit}

We use segment LCD in order to reduce power consumption. The driving of the segment LCD is different from that of ordinary liquid crystals, and an alternating voltage needs to be applied across its SEG and COM. At the same time, we abandoned the traditional driver chip and reduced the power consumption of the entire system. Because the 410 single-chip microcomputer does not have the ability to directly drive the segment LCD, we use resistor dividers to provide three states $(0, \mathrm{VCC} / 2, \mathrm{VCC})$ for the COM port [6]. This program can be used to directly drive the segment LCD with 410.

\section{CALCULATION}

\subsection{Calculation of operating current}

The CPU of this system turns on one AD sampling, two DA outputs, and direct drive segment LCD at its lowest frequency. The power consumption is about 1.5mA, LM358, HT7550, ILC7660 and other devices is about $0.5 \mathrm{~mA}$. The operating current of the whole system is below $2 \mathrm{~mA}$, which meets the requirements of micro-power industrial instrument's operating current below $4 \mathrm{~mA}$.

3.2 Calculation of the resistance of the liquid level detection circuit, the number of resistors and reed switches

The ADC of C8051F410 converts the voltage range from 0 to $2.2 \mathrm{~V}$. The output of DAC1 that we choose is $0.21 \mathrm{~mA}$. According to Ohm's law, the maximum resistance we need is $\mathrm{R}=U / I=8.8 K \Omega$. The designed detection circuit has a maximum range of $50 \mathrm{~cm}$, and its resolution depends on the arrangement density of the reed switch. On the premise that the number of reed switches can be arranged and the resolution meets the measurement requirements, we put a reed switch every $d=$ $50 \mathrm{~cm} / 88=0.56 \mathrm{~cm}$. Therefore, there are 88 resistances with a resistance of $100 \Omega$.

\subsection{Calculation of system measurement resolution}

The maximum sample voltage of the single-chip microcomputer 410 is $2.2 \mathrm{~V}$, and the DAC1 has a constant current of $0.25 \mathrm{~mA}$. Since 88 resistors of $100 \Omega$ are connected in series, the voltage is increased by $0.25 \mathrm{~mA}$ * $100 \Omega=25 \mathrm{mV}$ accordingly when the resistance of the liquid level detection circuit is more than one resistor in series. Since the sampling code value of the 12-bit AD of the 410 single-chip microcomputer is 0 to 4095, that is, when the liquid level detection circuit each has a series resistance, the sampling code value changes $\mathrm{X}=25 \mathrm{mV} * 4095 / 2.2 \mathrm{~V}=$ 46.534. When $0 \sim 4095$ is programmed to be converted to $0 \sim 50 \mathrm{~cm}$, the proportional coefficient that needs to be multiplied is $\mathrm{K}=50 / 4096=$ 0.01221 . Therefore, when the liquid level detection circuit is connected in series with one more resistor, the liquid level increase $\mathrm{L}=46.534$ * $0.01221=0.568 \mathrm{~cm}$. That is, the resolution of this system is $5.68 \mathrm{~mm}$, which can meet the general industrial level detection.

\subsection{Calculation of segment LCD refresh time}

The display module that we use is $5 \mathrm{~V}$ working voltage, $1 / 2$ bias, 4 duty segment LCD. If the display module is directly driven by the IO port of the microcontroller, the relationship between the refresh time and the execution time of other programs needs to be handled. The refresh rate of the segment $\mathrm{LCD}$ is $25 \sim 300 \mathrm{~Hz}$, and the general work is about $50 \mathrm{~Hz}$. The 4 duty segment LCD scans are performed four times. Each time the COM port is scanned one by one, and there is interval of $t=1 / 50=2 \mathrm{~ms}$ between each scan cycle [7]. For low power considerations, the microcontroller operates at the lowest frequency $(0.191406 \mathrm{MHz})$ and the 410-microcontroller's machine cycle is one clock cycle. So, its machine cycle is: $\mathrm{T}=1 / 0.191406=5.2$ us. The above value is put into the formula of the initial value of the timer: $65535-\mathrm{x} / \mathrm{T}=\mathrm{t}$, from which it can be obtained: $\mathrm{x}=65153$.

\section{SOFTWARE DESIGN}

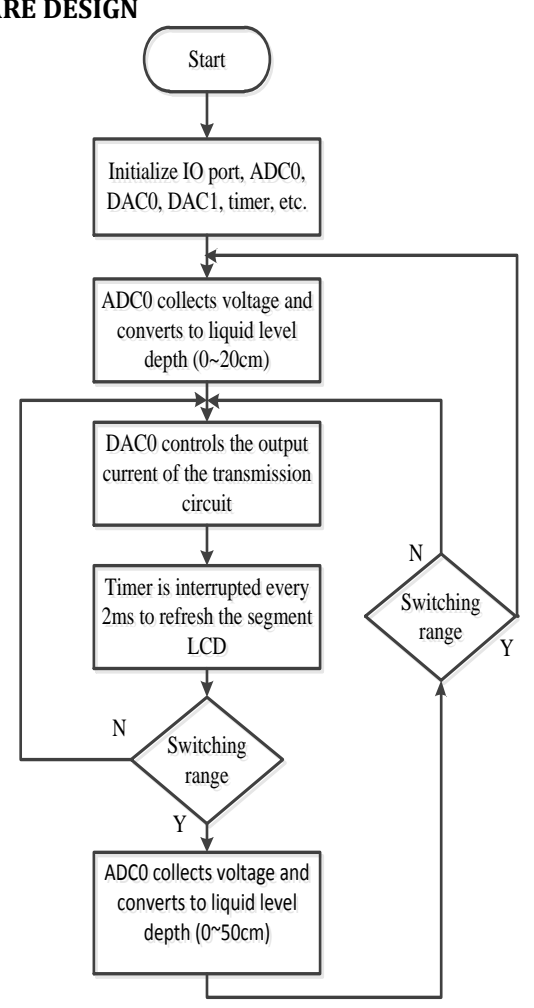

Figure 7: Program flow chart

The block diagram is shown in Figure 7. When the program begins to run, it firstly initializes the microcontroller's IO port, ADC0, timer, DAC0, opening the "constant current source" DAC1 and so on. When the program is running, ADC0 samples, and the collected voltage value is stored in the buffer. After filtering and conversion, the current liquid level depth and DAC0 output voltage are displayed by the segment LCD. Since the program for displaying is refreshed in the timer interrupt, real-time display of the liquid level change can be achieved. When the button for switching the range is pressed, the program will execute the corresponding subroutine of sampling and output according to the corresponding range of the 
button.

\section{EXPERIMENTAL TEST}

When the ranges are selected from $0 \sim 20 \mathrm{~cm}$ and $0 \sim 50 \mathrm{~cm}$, the points are measured at equal intervals. The data statistics table is shown in Table 1 , where $\mathrm{h} 1$ is the actual depth and $\mathrm{h} 2$ is the depth of measurement. The actual depth and measured depth of each point are given in the table.

Table 1: Measurement data

\begin{tabular}{|c|c|c|c|c|}
\hline Range & $\mathrm{h} 1 / \mathrm{cm}$ & $\mathrm{h} 2 / \mathrm{cm}$ & $\mathrm{h} 1 / \mathrm{cm}$ & $\mathrm{h} 2 / \mathrm{cm}$ \\
\hline \multirow[t]{5}{*}{$0 \sim 20 \mathrm{~cm}$} & 0.00 & 0.00 & 12.00 & 12.57 \\
\hline & 2.00 & 2.56 & 14.00 & 14.55 \\
\hline & 4.00 & 4.57 & 16.00 & 16.56 \\
\hline & 6.00 & 5.43 & 18.00 & 18.35 \\
\hline & 10.00 & 9.43 & 20.00 & 19.95 \\
\hline \multirow[t]{5}{*}{$0 \sim 50 \mathrm{~cm}$} & 5.00 & 5.56 & 30.00 & 29.47 \\
\hline & 10.00 & 10.55 & 35.00 & 35.55 \\
\hline & 15.00 & 15.57 & 40.00 & 39.46 \\
\hline & 20.00 & 20.57 & 45.00 & 44.43 \\
\hline & 25.00 & 25.55 & 50.00 & 49.99 \\
\hline
\end{tabular}

According to the test data, it can be seen that there are errors in the measurement results. We analyze the causes of the error and give the method to reduce the error as following:

According to the calculation results of the liquid level detection circuit part and the resolution part of the system, it can be obtained: Whenever the liquid level detection circuit is connected in series with a resistor, the sampling voltage is increased by $0.025 \mathrm{~V}$ accordingly. The sampling code value of the microcontroller ADC is increased by approximately 46.534 , and the liquid level is increased by approximately $0.568 \mathrm{~cm}$. If the resistor is not connected in series to the circuit (the reed switch is not conducting), the liquid level is reduced by $0.568 \mathrm{~cm}$ accordingly. The magnetic properties of the levitated ball cause the resistance value of the series-in circuit to be not very accurate. This causes voltage sampling error and eventually leads to a liquid level measurement error.

We also noticed that there was almost no measurement error when the liquid level was 0 or the highest range. Cause analysis: When the actual liquid level is $0 \mathrm{~cm}$, there is no conduction of the reed switch. That is, the resistance of the liquid level detection circuit is infinite, the sampling voltage is $0 \mathrm{~V}$, and the converted liquid level value is also $0 \mathrm{~cm}$. When the actual liquid level is full scale, the total resistance value is $8.8 \mathrm{~K} \Omega$, and the corresponding liquid level is the maximum range after conversion. So, there is almost no error when the liquid level is $0 \mathrm{~cm}$ or the highest range.

To reduce the error effectively, we could increase the number of resistors (that is, the arrangement density of the reed switches). Since the total resistance of the resistor is always $8.8 \mathrm{~K}$, the resistance of each resistor should be reduced. At this time, whenever the detection circuit is connected in series with one resistor, the corresponding added number in the sampling voltage will be reduced accordingly, and the final level error will be reduced [8].

\section{CONCLUSION}

This design uses C8051F410 microcontroller as the controller for voltage sampling and output, and the microcontroller directly drives the segment LCD as a display module. The liquid level analog quantity is converted into a $4 \sim 20 \mathrm{~mA}$ electrical signal by a transmission circuit and transmitted to the control room. The operating temperature of the system chip is wide temperature and suitable for most industrial applications. This system has low power consumption, simple peripheral circuits, flexible functions, easy commissioning and maintenance, and accurate measurement results. It is an innovation and an attempt. The test results can be concluded: when the liquid level is $0 \mathrm{~cm}$ or the highest range, the system has almost no error.
The resolution and accuracy of the system will be greatly improved when there are enough reeds. This method has certain reference function for the design of micro power consumption instrument.

\section{ACKNOWLEDGMENTS}

1. [201750244] Jilin Science and Technology Innovation Development Plan Project

2. [2018074] Science and technology research project of Jilin Institute of Chemical Technology

3. [2016033] Major science and technology projects in Jilin Institute of Chemical Technology

\section{REFERENCES}

[1] Issa, J. 2012. Display power analysis and design guidelines to reduce power consumption [J]. Journal of Information Display, 13 (4)

[2] Norman, T. 2012. How Electronic Access Control Systems Work [M]. Elsevier Inc.: 06-15.

[3] Peng, X.J. 2012. Design of an Ultra-Low Power Timer System Based on C8051f410[A]. Hong Kong Education Society. Proceedings of 2012 3rd International Conference on Information Technology for Manufacturing Systems (ITMS 2012 V236-237) [C]. Hong Kong Education Society, 5.

[4] Luecke, J. 2005. Signal Paths from Analog to Digital [M]. Elsevier Inc. 06-15.

[5] Li, B., Li, Z. 2018. Design of Automatic Monitoring System for Transfusion. Acta Electronica Malaysia, 2 (1), 07-10.

[6] Anonymous. 2011. NXP Debuts Cortex-M0 Microcontrollers with Integrated Segment LCD Drivers [J]. Wireless News.

[7] Yan, Z. 2018. Artificial Bee Colony Constrained Optimization Algorithm with Hybrid Discrete Variables and Its Application. Acta Electronica Malaysia, 2 (1), 18-20.

[8] Bloss, R. 2014. HARM type MEMS-based reed switch innovations address medical, automotive and other micro switching applications not previously possible [J]. Assembly Automation, 34 (3). 